Classification

Physics Abstracts

71.20

\title{
Etude de la structure électronique et magnétique de $\mathrm{CrO}_{2}$
}

\author{
S. Matar (*), G. Demazeau (*), J. Sticht, V. Eyert et J. Kübler \\ Technische Hochschule Darmstadt, Fachbereich Physik, D-6100 Darmstadt, Germany \\ (Reçu le 17 septembre 1991, accepté sous forme définitive le 14 novembre 1991)
}

\begin{abstract}
Résumé. - Les propriétés électroniques et magnétiques de $\mathrm{CrO}_{2}$ ont été déterminées par la méthode auto-cohérente de l'onde sphérique augmentée : A.S.W. Cette étude est menée dans le cadre d'une nouvelle approche visant à définir l'évolution de ses propriétés magnétiques à volume décroissant d'une part et à élucider les résultats expérimentaux récents de photoémission sur couches minces d'autre part. Les résultats des calculs montrent qu'une transition magnétique de type ferro $\Rightarrow$ antiferromagnétique est susceptible d'être induite sous l'effet de la pression. Les résultats expérimentaux pourraient être interprétés par une compression de l'octaèdre $\mathrm{CrO}_{6}$ au sein de la maille.
\end{abstract}

\begin{abstract}
The electronic and magnetic properties of $\mathrm{CrO}_{2}$ were investigated using the selfconsistent A.S.W. method in a new approach to study the evolution of its magnetic properties at decreasing volume and to assess recent photoemission results on thin films. The results show that a magnetic transition of ferro $\Rightarrow$ antiferromagnetic type is likely to be induced under pressure. Experimental results could be explained by a compression of the $\mathrm{CrO}_{6}$ octahedron within the cell.
\end{abstract}

\section{Introduction.}

Le dioxyde de chrome, l'un des rares oxydes ferromagnétiques à température ambiante, a de par ses propriétés particulières suscité beaucoup d'intérêt depuis sa découverte $[1,3]$.

Malgré l'existence de nombreux travaux théoriques sur les oxydes isostructuraux tels que $\mathrm{IrO}_{2}$ et $\mathrm{RuO}_{2}$ relatifs au calcul de leurs structures électroniques dès les années 1970 [4], le premier calcul similaire de $\mathrm{CrO}_{2}$ n'a été publié qu'en 1986 [5]. Les résultats obtenus soulignent le caractère semi-métallique de $\mathrm{CrO}_{2}$. i.e. que la densité d'états des spins majoritaires a un caractère métallique alors que la densité d'états des spins minoritaires fait apparaître un gap de $\cong 2 \mathrm{eV}$ entre les bandes $\mathrm{p}$ de l'oxygène situées en dessous du niveau de Fermi $\left(E_{\mathrm{F}}\right)$ et les bandes d du chrome situées en dessus de $E_{\mathrm{F}}$ avec pour conséquence une polarisation de $100 \%$ des spins. Le moment de $2 \mu \mathrm{B}$ par unité formulaire $\mathrm{CrO}_{2}$ est en accord avec 1'expérience [1].

La première étude expérimentale de la structure électronique de $\mathrm{CrO}_{2}$ a été effectuée en 1976 sur poudre par Ikemoto et al. [6]. L'analyse du spectre de photoémission X \{X.P.S.\} dans la région de la bande de valence fait apparaître une densité d'état finie au

$\left.{ }^{*}\right)$ Adresse permanente : L.C.S.-C.N.R.S., Université Bordeaux 1, 33405 Talence Cedex, France. 
niveau de Fermi soulignant ainsi le caractère métallique de l'oxyde. La structure de bande a été analysée dans le cadre du modèle phénoménologique développé par Goodenough [7]. En 1987 une étude de couches minces polycristallines de cet oxyde sur substrats $\mathrm{RuO}_{2}, \mathrm{TiO}_{2}$ (ou $\mathrm{Al}_{2} \mathrm{O}_{3}$ ) a été effectuée au moyen de la spectroscopie de photoémission à polarisation de spin (spin-resolved photoemission spectroscopy) [8]. Les résultats obtenus montrent une polarisation de $100 \%$ des spins - en accord avec le calcul [5] — cependant ce phénomène n'est observé qu'à $2 \mathrm{eV}$ en dessous du niveau de Fermi. En contradiction avec le calcul et avec le caractère de conducteur métallique de $\mathrm{CrO}_{2}$ confirmé par Ikemoto et al. [6], les auteurs concluent que $E_{\mathrm{F}}$ ne traverse pas les états $3 \mathrm{~d}$ du chrome.

Compte tenu des divergences entre les résultats des travaux antérieurs visant à déterminer la structure électronique de $\mathrm{CrO}_{2}$, ils nous a paru important de la repréciser dans une nouvelle approche.

\section{Considérations sur la structure de $\mathrm{CrO}_{2}$.}

L'oxyde $\mathrm{CrO}_{2}$ cristallise dans la structure de type rutile (groupe d'espace $\mathrm{P}_{4_{2} / \mathrm{mnm}}$ ) avec deux groupements formulaires par maille (Fig. 1). Les atomes de chrome occupent les positions (2a) : $0,0,0$ et $\frac{1}{2}, \frac{1}{2}, \frac{1}{2}$; les atomes d'oxygène se plaçant dans les positions particulières (4f): $\pm x, \pm x, 0 ; \pm x+\frac{1}{2}, \mp x+\frac{1}{2}, \frac{1}{2}$. Plusieurs travaux ont été consacrés à la détermination précise des paramètres de $\mathrm{CrO}_{2}$ [9-11]. Alors que la plupart d'entre eux sont en bon accord concernant les paramètres $a$ et $c$ (Tab. I), une disparité est observée pour les valeurs de $x_{(4 f)}$ déterminant la position de 1'oxygène. Or dans $\mathrm{CrO}_{2}$ l'environnement du chrome n'est pas un octaèdre parfait : il est en fait caractérisé par deux types de distances :

$$
\begin{aligned}
& d_{1}=\sqrt{2} \cdot x \cdot a \\
& d_{2}=\left|2\left(\frac{1}{2}-x\right)^{2}+\left(\frac{c}{2 a}\right)^{2}\right|^{1 / 2} a .
\end{aligned}
$$

Il en résulte qu'un octaèdre $\mathrm{MO}_{6}$ régulier est obtenu par l'égalité $(1)=(2)$ résolue pour $x$; d'où

$$
x=\frac{1}{4}+\frac{c^{2}}{8 a^{2}}
$$

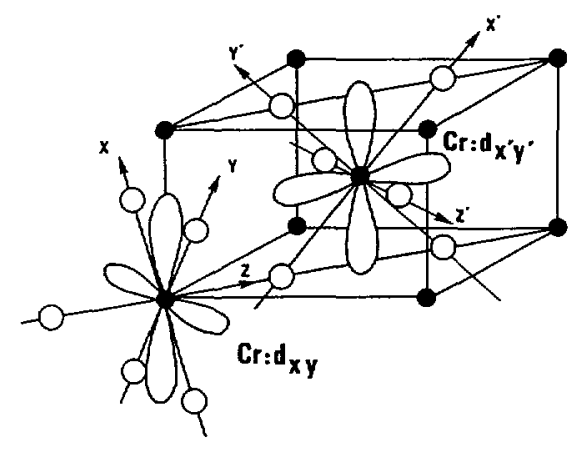

Fig. 1. - Structure cristalline de $\mathrm{CrO}_{2}$. Sphères noires : $\mathrm{Cr}$; sphères blanches : $\mathrm{O}$.

[Crystal structure of $\mathrm{CrO}_{2}$. Black spheres : $\mathrm{Cr}$; white spheres : $\left.\mathrm{O}.\right]$ 
Les valeurs de $x$ obtenues pour $\mathrm{CrO}_{2}$ et différents oxydes de structure rutile sont données au tableau I. Il est intéressant de noter que l'examen des valeurs de $x_{(4 f)}$ ne permet pas de conclure si l'octaèdre est allongé ou comprimé.

Tableau I. - Données cristallographiques sur $\mathrm{CrO}_{2}$ et autres oxydes de structure rutile. [Crystallographic data on $\mathrm{CrO}_{2}$ and other rutile-type oxides.]

\begin{tabular}{|c|c|c|c|c|c|}
\hline $\mathrm{MO}_{2}$ & $a \AA$ & $c / a$ & $x(4 f)$ & $\underset{(4 \mathrm{f})}{x\left(d_{1}=d_{2}\right)}$ & $d_{1} / d_{2}$ \\
\hline $\begin{array}{c}\mathbf{M}=\mathbf{C r} \\
{[9]}\end{array}$ & 4,421 & 0,64958 & 0,3053 & 0,304 & 1,005 \\
\hline $\begin{array}{c}\mathrm{M}=\mathrm{Cr} \\
{[10]}\end{array}$ & 4,421 & 0,65981 & $\begin{array}{r}0,301 \\
\pm 0,004\end{array}$ & 0,304 & 0,982 \\
\hline $\begin{array}{c}\mathrm{M}=\mathrm{Cr} \\
{[11]}\end{array}$ & 4,4190 & 0,65974 & 0,3026 & 0,304 & 0,990 \\
\hline $\begin{array}{c}\mathrm{M}=\mathrm{Ru} \\
{[4]}\end{array}$ & 4,492 & 0,696 & 0,306 & 0,310 & 0,980 \\
\hline $\begin{array}{c}\mathrm{M}=\mathrm{Os} \\
{[4]}\end{array}$ & 4,500 & 0,708 & 0,308 & 0,313 & 0,977 \\
\hline $\begin{array}{c}\mathrm{M}=\mathrm{Ir} \\
{[4]}\end{array}$ & 4,498 & 0,701 & 0,307 & 0,312 & 0,981 \\
\hline
\end{tabular}

\section{Méthodologie des calculs.}

Les calculs ont été effectués - comme indiqué dans la référence [5] — par la méthode A.S.W. (augmented spherical wave) auto-cohérente [12]. Dans cette étude la version relativiste scalaire [13] a été utilisée. Cette méthode est basée sur l'approximation de la sphère atomique (A.S.A.) qui impose la condition :

$$
V_{\text {maille }}=\sum V_{\text {sphères atomiques }}
$$

avec pour conséquence la prise en compte de tout le volume dans la résolution de l'équation de Schrödinger monoélectronique. En outre, dans la mesure où l'espace interatomique - qui peut varier selon la compacité et être assez important comme dans $\mathrm{CrO}_{2}$ - est rempli par des pseudo-atomes avec $Z=0$ appelés «sphères vides»(ES), l'erreur du non-traitement de l'espace interstitiel - traité explicitement dans d'autres méthodes similaires - est réduite. Ces sphères contenant les « queues » (i.e. « résidus » en termes de densités de charges) des fonctions d'onde sphériques des atomes voisins, il y en résulte une accumulation de charges. Il faut noter cependant que la condition (3) implique un certain recouvrement des sphères atomiques conduisant à des erreurs dans cette région — d'où l'approximation de sphères atomiques : A.S.A. Ainsi le choix des rayons des sphères n'est pas unique, ceux-ci peuvent être optimisés en recherchant une configuration à laquelle correspond un minimum d'énergie. 
Ainsi en plus du Cr en (2a) et de l'oxygène en (4f) des ES ont été introduites en (4c) : 0 , $\frac{1}{2}, 0 ; \frac{1}{2}, 0, \frac{1}{2} ; 0, \frac{1}{2}, \frac{1}{2} ; \frac{1}{2}, 0,0\left(\mathrm{ES}_{1}\right)$ et en $(4 \mathrm{~g}): \pm x, \mp x, 0 ; \frac{1}{2} \pm x, \frac{1}{2} \pm x$, $\frac{1}{2}\left(\mathrm{ES}_{2}\right)$.

Cet agencement de la maille en vue des calculs A.S.W. ainsi que le choix de rayons égaux pour les sphères de $\mathrm{Cr}$ et $\mathrm{O}$ sont identiques à ceux adoptés dans les calculs antérieurs [5]. Une différence par rapport à ces calculs qui utilisent une sphère de 1,9 a.u. (1 a.u. = $0,529 \AA$ ) pour $\operatorname{ES}_{1}(4 \mathrm{c})$ et une de 1,2 a.u. pour $\mathrm{ES}_{2}(4 \mathrm{~g})$ réside dans notre choix de sphères de rayons égaux pour les deux ensembles de sphères vides avec un rapport optimisé $r(\mathrm{Cr}, \mathrm{O}) / r(\mathrm{ES})=1,316$ et des rayons intermédiaires des valeurs données ci-dessus. Le critère à l'origine de ce choix est celui d'éviter des sphères vides de grand rayon qui seraient susceptibles de drainer une forte densité de charges. Un tel comportement donnant à ces sphères un caractère atomique rendrait leur signification non physique.

La base de fonctions d'ondes pour chaque espèce était : $\mathrm{Cr}: \mathrm{s}, \mathrm{p}, \mathrm{d}(\boldsymbol{\ell}=0,1,2), \mathrm{O}: \mathrm{s}, \mathrm{p}$ $(\ell=0,1)$ et ES : $s(\ell=0)$ comme base explicite avec une extension à $\ell_{\max }+1$ pour les résidus de charges.

\section{Etude de la structure électronique et magnétique de $\mathrm{CrO}_{2}$.}

4.1 RECHERCHE DES CONDITIONS D'ÉQUILIBRE (ÉNERGIE, VOlUME) DE $\mathrm{CrO}_{2}$. - Un des apports majeurs des calculs de structure électronique et magnétique des matériaux est de permettre de déterminer leurs propriétés d'équilibre (volume, énergie) au travers de calculs effectués à volumes variables voisins de la valeur expérimentale. L'intérêt d'une telle étude est de pouvoir être entreprise pour les trois différents états magnétiques qu'un système peut - ou ne peut pas - adopter à $0 \mathrm{~K}$, soit : ferro- (F), antiferro- (AF) et non magnétiques (NM) et de permettre de conclure quant à leurs stabilités relatives. La valeur du volume à l'équilibre est alors celle qui corespond à un minimum d'énergie totale — variationnelle.

Cette étude a été appliquée à $\mathrm{CrO}_{2}$ suivant deux approches différentes. Du fait de la symétrie du réseau quadratique de $\mathrm{CrO}_{2}$ la variation du volume peut être isotrope (rapport $c / a$ constant) ou anisotrope (variation de $c / a$ ). Une variation anisotrope $\mathrm{du}$ volume trouve sa justification dans le schéma phénoménologique que Goodenough [7] donne des interactions magnétiques au sein de $\mathrm{CrO}_{2}$ en relation avec le réseau. En effet en dessous de la température de Curie $\left(125^{\circ} \mathrm{C}\right)$ la maille rutile adopte un rapport cla anormalement élevé $\left(0,66 \mathrm{au}\right.$ lieu de $\left.(c / a)_{\mathrm{TiO}_{2}}=0,64\right)$ afin de minimiser les interactions AF entre les orbitales à demi-remplies le long de l'axe $c$ permettant ainsi de préserver l'ordre ferromagnétique du système. Ainsi la recherche des propriétés à l'équilibre de $\mathrm{CrO}_{2}$ a-t-elle été entreprise selon ces deux voies pour les configurations $\mathrm{F}$ et $\mathrm{AF}$.

4.1.1 Diminution isotrope du volume. - Le paramètre calculé à l'équilibre pour une configuration $F$ est de $4,313 \AA$. Cette valeur inférieure d'environ $2,5 \%$ à la valeur expérimentale $(4,421 \AA)$ montre un bon accord. Il est à noter néanmoins que les valeurs des paramètres à l'équilibre obtenues à partir des calculs de structure de bande sont systématiquement inférieures aux valeurs expérimentales. Ceci est inhérent à l'approximation de la densité locale de spin utilisée par la méthode ASW et illustre une de ses insuffisances.

La configuration AF a été simulée en contraignant les spins en $0,0,0$ et $\frac{1}{2}$, $\frac{1}{2}, \frac{1}{2}$ à s'aligner antiparallèlement d'où la succession de plans de spins opposés perpendiculairement à l'axe $c$. Le paramètre à l'équilibre ainsi calculé est de 4,302 $\AA$, inférieur à celui de la 
configuration $\mathrm{F}$. La faible différence de volume entre les deux configurations est accompagnée d'une faible différence d'énergie (l'état $F$ est de 0,02 Ryd. (1 Ryd. = 13,6 eV) plus stable que l'état AF). En outre les valeurs de module volumique (inverse de la compressibilite) sont de 2660 et 2590 kbar respectivement pour les deux configurations. Ces résultats mettent en évidence la proximité des deux configurations et suggèrent la possibilité d'une transition $\mathrm{F} \Rightarrow \mathrm{AF}$ par réduction du volume - en particulier par application de la pression. Cependant le tracé des courbes d'évolution de l'énergie variationnelle $E$ en fonction du volume : $E=\mathrm{f}(V)$ (Fig. 2) pour les deux configurations magnétiques montre que les courbes $\mathrm{F} 1$ et AF1 (ferro- et antiferro- respectivement) ne se croisent pas, au moins dans la gamme de volumes considérée. Physiquement ceci signifie qu'une compression - c'est-à-dire une réduction de volume - ne permet pas d'obtenir une transition magnétique.

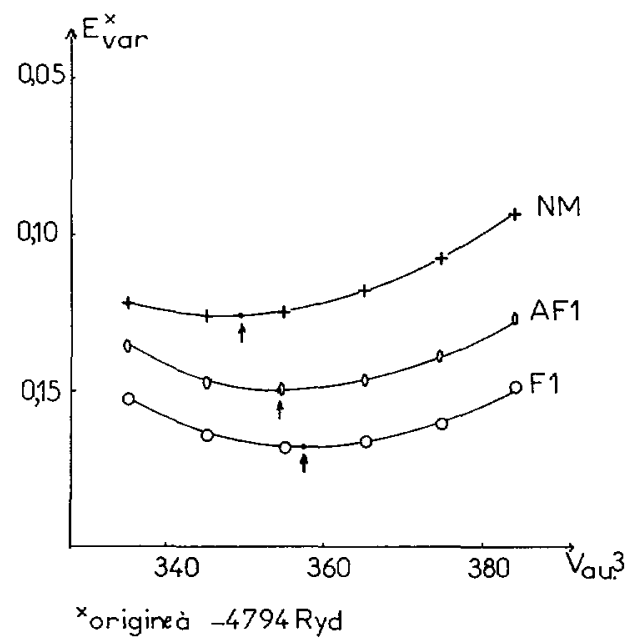

Fig. 2. - Evolution de l'énergie variationnelle totale en fonction d'une variation isotrope du volume pour les trois états magnétiques de $\mathrm{CrO}_{2}$. Les flèches verticales indiquent les valeurs d'équilibre $\left(E_{\text {eq }}, V_{\text {eq }}\right)$. L'origine des énergies est à -4794 Ryd.

[Evolution of total variational energy as a function of an isotropic variation of the volume for the three magnetic states of $\mathrm{CrO}_{2}$. Vertical arrows indicate the values at equilibrium $\left(E_{\mathrm{eq}}, V_{\mathrm{eq}}\right)$. The origin of the energies is at -4794 Ryd.]

Le calcul du matériau $\mathrm{CrO}_{2}$ dans un état non magnétique a été également mené. Il a permis d'obtenir pour les valeurs à l'équilibre un volume plus faible que celui de l'état AF avec un paramètre $a_{\mathrm{eq}}(\mathrm{NM})=4,279 \AA$. En outre les différences d'énergies ont pour valeurs respectives : $E_{\text {eq }}(\mathrm{AF})-E_{\mathrm{eq}}(\mathrm{NM})=-0,02$ Ryd. et $E_{\mathrm{eq}}(\mathrm{F})-E_{\mathrm{eq}}(\mathrm{NM})=-0,03 \mathrm{Ryd}$. Malgré les faibles différences entre les valeurs $E$ et $V$ à l'équilibre, on n'observe pas de croisement entre les différentes courbes. Il faut noter que les résultats obtenus mettent en jeu de faibles variations d'énergie et devraient être considérés avec prudence. La diminution de la valeur du volume d'équilibre dans la séquence $\mathrm{F} \Rightarrow \mathrm{AF} \Rightarrow \mathrm{NM}$ met en évidence l'effet de la diminution de la pression magnétique interne du matériau.

4.1.2 Diminution anisotrope du volume. - La diminution anisotrope du volume introduit des erreurs supplémentaires au niveau de l'A.S.A. En effet le recouvrement des sphères atomiques et $\mathrm{ES}$ - n'est plus isotrope - étant donné que la diminution de leurs volumes a été effectuée dans les mêmes conditions que plus haut. Afin de réduire cette erreur il a été 
nécessaire de représenter les variations des différences d'énergies totales entre l'état $F$ et $\mathrm{AF}$ d'une part et l'état NM d'autre part. Les courbes F2 et AF2 de la figure 3 montrent la variation de $\Delta E$ (différence d'énergie : $\Delta E=E_{\mathrm{F} \text { ou } \mathrm{AF}}-E_{\mathrm{NM}}$ ) en fonction du volume pour les états $\mathrm{F}$ et $\mathrm{AF}$ dans cette hypothèse. L'évolution de ces courbes montre un comportement différent de celui de la figure 2 : alors que ces dernières montrent un minimum qui a permis de calculer les paramètres à l'équilibre, F2 et AF2 ne montrent pas de minimum et présentent une pente plus prononcée indiquant une déstabilisation du système dans de telles conditions ; effet dû en partie à l'A.S.A. utilisée. Ceci mène à formuler une certaine réserve quant au sens physique des résultats obtenus. Il est néanmoins intéressant de noter que les deux courbes se croisent à un volume de $354 \mathrm{a} . \mathrm{u}^{3}$. voisin des volumes à l'équilibre des états F et AF (357 et 355 a.u. respectivement - courbes F1 et AF1). Cette observation qui est en accord avec le schéma de Goodenough [7] semble être en faveur d'une transition magnétique lorsqu'un monocristal de $\mathrm{CrO}_{2}$ est comprimé sélectivement suivant l'axe $c$.

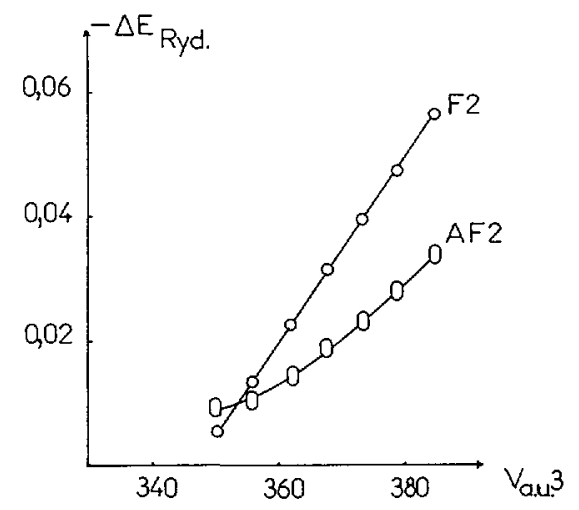

Fig. 3. - Evolution des différences d'énergies $\Delta E=E_{\mathrm{Fou} \mathrm{AF}}-E_{\mathrm{NM}}$ en fonction d'une variation anisotrope du volume de $\mathrm{CrO}_{2}$.

[Evolution of the energy differences $\Delta E=E_{\mathrm{F} \mathrm{or} A F}-E_{\mathrm{NM}}$ as a function of an anisotropic variation of the volume of $\mathrm{CrO}_{2}$.]

4.2 ETUde DE $\mathrm{CrO}_{2}$ AUX PARAMÈTRES EXPÉRIMENTAUX. - Cette étude a été entreprise en considérant les paramètres $a$, cla et $x_{(4 \mathrm{f})}$ de Thamer et al. [9] utilisés par Schwarz [5].

Afin de permettre une comparaison avec les travaux antérieurs, deux calculs ont été effectués avec pour unique différence le choix des rayons des sphères vides (cf. Sect. 2). Les calculs spin-polarisés (magnétiques) ont été effectués avec 126 points $\mathrm{k}$ dans la représentation irréductible de la zone de Brillouin soit $1 / 16^{\mathrm{e}}$ de la zone entière. Le nombre de points $\mathrm{k}$ a été augmenté progressivement au cours des cycles itératifs jusqu'à ce qu'aucune variation des moments magnétiques et de l'énergie totale ne soit observée. Pour les deux ensembles de calculs les charges locales partielles (états $s, p$ et d) pour les deux directions de spins ainsi que les moments magnétiques sur chaque site sont donnés au tableau II ( $\mathrm{a}$ et $\mathbf{b}$ ).

4.2.1 Moments magnétiques. - Le moment du chrome voisin de $2 \mu \mathrm{B}$ provient en majeure partie de la différence d'occupation des états $3 d$ entre les deux directions de spin. Les occupations des états «spin-up » restant majoritaires la polarisation reste positive d'où le signe du moment du chrome. Cette tendance est inversée pour le moment de l'oxygène qui est négatif. Le moment total par maille est de $4 \mu \mathrm{B}$. Ce nombre est entier du fait de la présence d'un gap dans les densités d'états des spins minoritaires dont le nombre est par conséquent 
entier. Il en résulte que le nombre de spins majoritaires est entier lui aussi puisque la maille doit rester neutre :

$$
N_{\text {TOTAL-MAILLE }}=N_{\text {sp./maj. }}+N_{\text {sp./min. }} .
$$

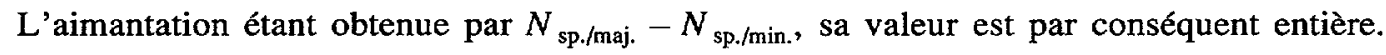

4.2.2 Distributions des charges. - Les valeurs des charges totales au niveau de Fermi $\left(Q_{\text {tot }}\right)$ montrent que le transfert de charges se produit depuis les sphères du chrome à celles de l'oxygène d'une part et à l'intérieur des sphères $\mathrm{ES}_{1}$ et $\mathrm{ES}_{2}$ d'autre part. Pour ces dernières les valeurs consignées au tableau IIa montrent que le transfert est proportionnel à leurs distances respectives à l'oxygène et au chrome, les distances les plus courtes étant : $d_{\mathrm{ES}_{1}-\mathrm{O}}=1,60 \AA$ et $d_{\mathrm{ES}_{2}-\mathrm{Cr}}=1,91 \AA$ alors que $d_{\mathrm{ES}_{2}-\mathrm{O}}=2,70 \AA$ et $d_{\mathrm{ES}_{1}-\mathrm{Cr}}=2,21 \AA$. Ainsi la majeure contribution de charges dans $E S_{2}$ provient du chrome, celle dans $E S_{1}$ provenant de l'oxygène. La charge supérieure dans $\mathrm{ES}_{2}$ provient de sa proximité au chrome. Malgré les volumes égaux des deux sphères vides, la charge de la sphère $E_{1} S_{1}$ est plus faible ; ceci étant dû à un transfert en retour i.e. dans le sens $E S_{1} \Rightarrow 0$ alors que pour $E S_{2}$ le transfert se produit dans le sens $\mathrm{Cr} \Rightarrow \mathrm{ES}_{2}$. Ceci est différent des résultats dans le tableau II où la tendance pour

Tableau II. - Charges partielles locales pour les spins majoritaires ( $\uparrow$ ) et les spins minoritaires $(\downarrow)$ dans $\mathrm{CrO}_{2}$. Calculs effectués aux paramètres expérimentaux: $a=8,35448$ a.u. ; cla $=0,65958$. a) Ce travail. b) Calculs selon données de la référence [5]. [Local partial charges for majority $(\uparrow)$ and minority $(\downarrow)$ spins of $\mathrm{CrO}_{2}$. Calculation performed with the experimental lattice constants : $a=8.35448$ a.u. ; $c / a=0.65958$. a) This work. b) Calculations according to reference [5].]

a)

\begin{tabular}{|c|c|c|c|c|c|c|c|c|c|}
\hline $126 \mathrm{k}$ & $r_{\mathrm{Ws}}$ a.u. & $n_{\mathrm{s}} \uparrow$ & $n_{\mathrm{s}} \downarrow$ & $n_{\mathrm{p}} \uparrow$ & $n_{\mathrm{p}} \downarrow$ & $n_{\mathrm{d}} \uparrow$ & $n_{\mathrm{d}} \downarrow$ & $Q_{\text {Tot. }}$ & $M_{\mathrm{at}} \mu \mathrm{B}$ \\
\hline $\mathrm{Cr}$ & 2,12963 & 0,11 & 0,10 & 0,17 & 0,16 & 2,75 & 0,72 & 4,09 & 2,049 \\
\hline $\mathrm{O}$ & 2,12963 & 0,89 & 0,89 & 2,21 & 2,27 & 0,04 & 0,01 & 6,26 & $-0,034$ \\
\hline $\mathrm{ES}_{1}$ & 1,61772 & 0,07 & 0,07 & 0,06 & 0,06 & - & - & 0,26 & 0,001 \\
\hline $\mathrm{ES}_{2}$ & 1,61772 & 0,10 & 0,10 & 0,09 & 0,08 & - & - & 0,37 & 0,008 \\
\hline
\end{tabular}

b)

\begin{tabular}{|c|c|c|c|c|c|c|c|c|c|}
\hline $126 \mathrm{k}$ & $r_{\mathrm{Ws}}$ a.u. & $n_{\mathrm{s}} \uparrow$ & $n_{\mathrm{s}} \downarrow$ & $n_{\mathrm{p}} \uparrow$ & $n_{\mathrm{p}} \downarrow$ & $n_{\mathrm{d}} \uparrow$ & $n_{\mathrm{d}} \downarrow$ & $Q_{\text {Tot. }}$ & $M_{\mathrm{at}} \mu \mathrm{B}$ \\
\hline $\mathrm{Cr}$ & 2,38 & 0,11 & 0,10 & 0,17 & 0,16 & 2,78 & 0,77 & 4,16 & 2,032 \\
\hline $\mathrm{O}$ & 2,38 & 0,89 & 0,89 & 2,19 & 2,24 & 0,04 & 0,02 & 6,26 & $-0,023$ \\
\hline $\mathrm{ES}_{1}$ & 1,9 & 0,10 & 0,10 & 0,11 & 0,11 & 0,06 & 0,06 & 0,53 & 0,005 \\
\hline $\mathrm{ES}_{2}$ & 1,2 & 0,04 & 0,04 & 0,02 & 0,02 & - & - & 0,13 & 0,002 \\
\hline
\end{tabular}


les sphères vides est inversée et pourrait s'expliquer par le choix des rayons des sphères vides [5]. Le rayon de 1,9 a.u. pour $\mathrm{ES}_{1}$ a permis un plus grand recouvrement avec la sphère du chrome et donc d'y permettre un plus grand transfert $(0,53 \mathrm{e})$ au détriment de la sphère $\mathrm{ES}_{2}$ de 1,2 a.u. $(0,13 \mathrm{e})$. Il est intéressant de noter que le rapport des volumes de ces sphères vides est égal au rapport des charges dans ces sphères : soit $\cong 4$.

4.2.3 Densités d'états. - Pour le calcul utilisant deux sphères vides de rayons égaux, les densités d'états (DOS) totales pour chaque direction de spin ainsi que les bandes correspondantes sont montrées à la figure 4. Les énergies sont comptées par rapport à une origine située au niveau de Fermi : $E_{\mathrm{F}}\left(\mathrm{CrO}_{2}\right)=9,14 \mathrm{eV}$. De bas en haut les DOS sont attribuées aux états $2 \mathrm{~s}$ et $2 \mathrm{p}$ de l'oxygène puis aux états $3 \mathrm{~d}$ du chrome. Ceci est rendu plus explicite par les DOS projetées aux sites du chrome, de l'oxygène et des sphères vides montrées à la figure 5 .

La figure 5a montre que les DOS à majorité de spin du chrome sont traversées par le niveau de Fermi. La bande 3d présente un aspect de bande étroite avec un certain caractère localisé comme le visualise le pic pointu au-dessous de $E_{\mathrm{F}}$. En revanche pour les spins minoritaires $E_{\mathrm{F}}$ se trouve dans un gap avec une bande $3 \mathrm{~d}$ vide impliquant un caractère semi-métallique de $\mathrm{CrO}_{2}[5]$.

4.2.4 Structure de bandes. - L'examen de la structure de bande pour les deux états de spin majoritaires et minoritaires - permet au travers d'un simple comptage des bandes de retrouver le moment total par maille i.e. $4 \mu \mathrm{B}$. En effet pour chacun des deux $\mathrm{Cr}$ il y a à l'état neutre $5 \mathrm{e}^{-} 3 \mathrm{~d}$ avec les deux possibilités de spin : en tout il y a donc 20 états décrits dans 20 bandes. Pour les états à minorité de spin (Fig. 4b), les bandes d vides peuvent être comptées au-dessus de $E_{\mathrm{F}}$ soit 10 bandes. Il reste 10 bandes pour les spins majoritaires (Fig. 4a) : on peut compter au total 6 bandes au-dessus de $E_{\mathrm{F}}$ donc vides. Ainsi reste-t-il audessous de $E_{\mathrm{F}} 4$ bandes pleines pour les deux $\mathrm{Cr}$. Par suite 2 bandes pleines correspondent à chaque $\mathrm{Cr}$, chacune ayant un électron, donc deux électrons donnant un moment total de $2 \mu \mathrm{B}$ par unité formulaire de $\mathrm{CrO}_{2}$. Malgré la proximité de cette valeur de celle qu'aurait donnée un ion $d^{2}$ i.e. $\mathrm{Cr}^{4+}$, il faut noter que ce moment provient de l'addition des moments de tous les constituants de la maille.

4.3 DisCuSSION DES RÉSUltats. - Le schéma généralement retenu pour la structure de bande de $\mathrm{CrO}_{2}$ est celui d'un oxyde ionique proposé par Goodenough [7] : le moment magnétique de $2 \mu \mathrm{B}$ serait dû d'une part à un électron localisé dans une orbitale $t_{2 \mathrm{~g}}$ (e.g. $\mathrm{d}_{x y}$ dans le cas de l'aplatissement de l'octaèdre $\mathrm{CrO}_{6}$ ), d'autre part à un électron délocalisé mais fortement corrélé dans une bande étroite formée des autres orbitales $\mathrm{t}_{2 \mathrm{~g}}$ soit $\mathrm{d}_{x z}$ et $\mathrm{d}_{y z}$. Ce schéma est montré à la figure 6. Malgré le fait que les calculs effectués ne permettent pas de montrer pour les DOS (Fig. 5) une telle représentation, il est intéressant de signaler quelques similitudes avec le schéma de la figure $6:$ pour les états $3 \mathrm{~d}$ à majorité de spins du chrome (Fig. 5a), le pic pointu en dessous de $E_{\mathrm{F}}$ correspondrait à l'état localisé $\left(t_{2 g \alpha}^{\|}\right)$contenant un spin, le pic plus large entourant ce pic contiendrait l'autre spin et correspondrait à la bande étroite $\pi_{\alpha}^{*}$.

4.3.1 Examen de la structure de bandes. - Etant donné l'approximation de la sphère atomique utilisée, la discussion du caractère de la liaison chimique au sein de $\mathrm{CrO}_{2}$ doit tenir compte d'un point essentiel : la nature de la liaison chimique ne peut être clairement déterminée par suite du partage de la maille en sphères non physiques. Le décompte des électrons à l'intérieur de ces sphères n'est donc pas une indication pouvant déterminer si la liaison a un caractère ionique ou covalent prédominant. 
A l'examen de la structure de bande pour les états à minorité de spins, une séparation entre états $\mathrm{d}$ vides du chrome et états $\mathrm{p}$ pleins de l'oxygène est observée avec un gap de $2 \mathrm{eV}$ (Fig. 4b). Par suite une tendance vers un caractère ionique de la liaison pourrait être suggéré. En revanche pour les états à majorité de spins une telle séparation est moindre (Fig. 4a) et semble en faveur d'un «mélange » des états de l'oxygène et du chrome, d'où une tendance vers un caractère plus covalent de la liaison. Cette différence de comportement semble avoir
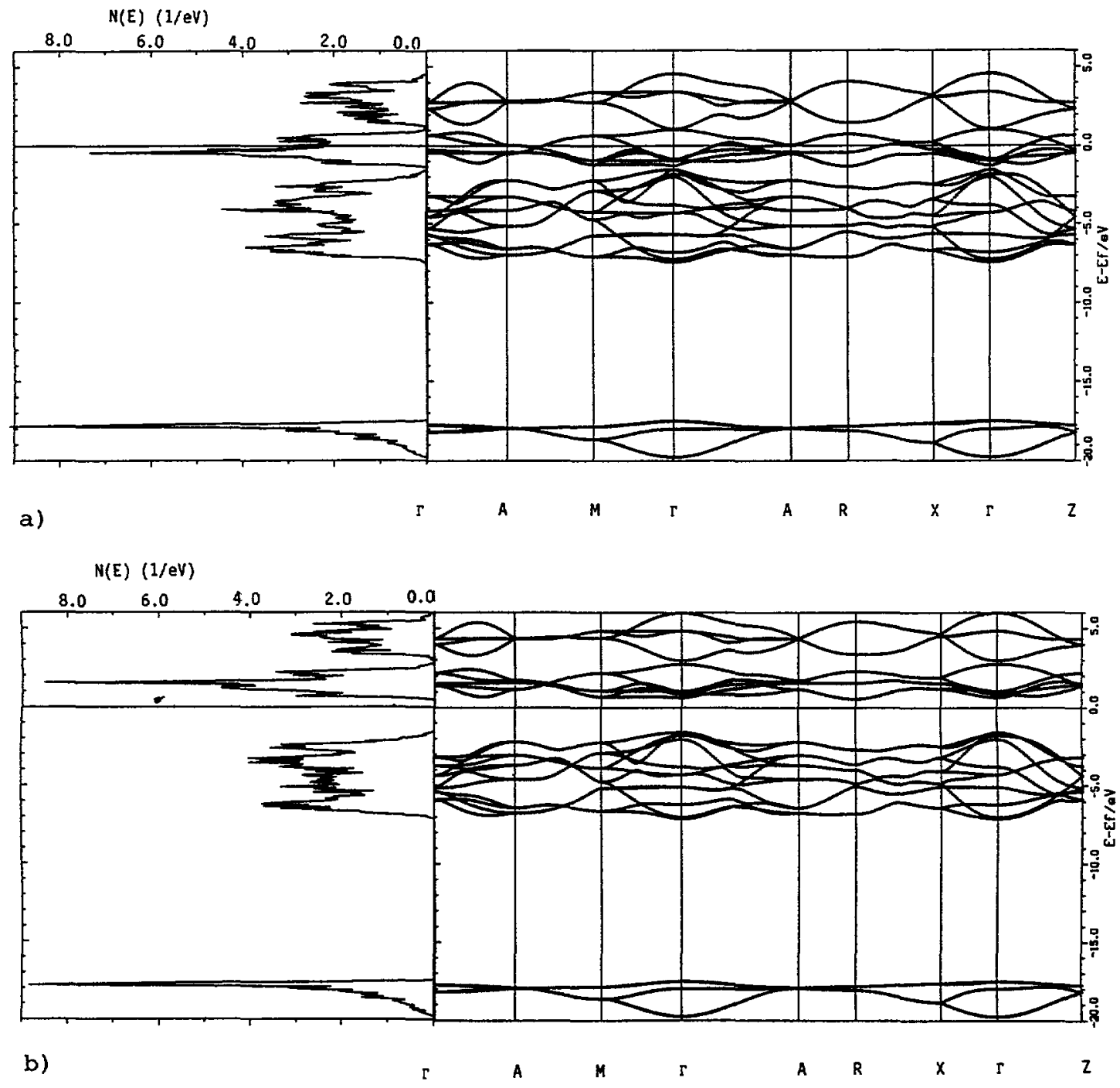

Fig. 4. - Variations des densités d'états (DOS) - partie de gauche - et des bandes d'énergie dans la première zone de Brillouin - partie de droite - en fonction de l'énergie pour $\mathrm{CrO}_{2}$ suivant les deux directions de spins : spins majoritaires a) et spins minoritaires b). L'origine des énergies est au niveau de Fermi i.e. à $9,14 \mathrm{eV}$.

[Variations of the densities of states (DOS) - left hand side - and of the energy bands in the first Brillouin zone - right hand side - as a function of energy for $\mathrm{CrO}_{2}$ along the two spin directions : majority spins a); minority spins b). The origin of the energies is at Fermi level i.e. at $9.14 \mathrm{eV}$.] 


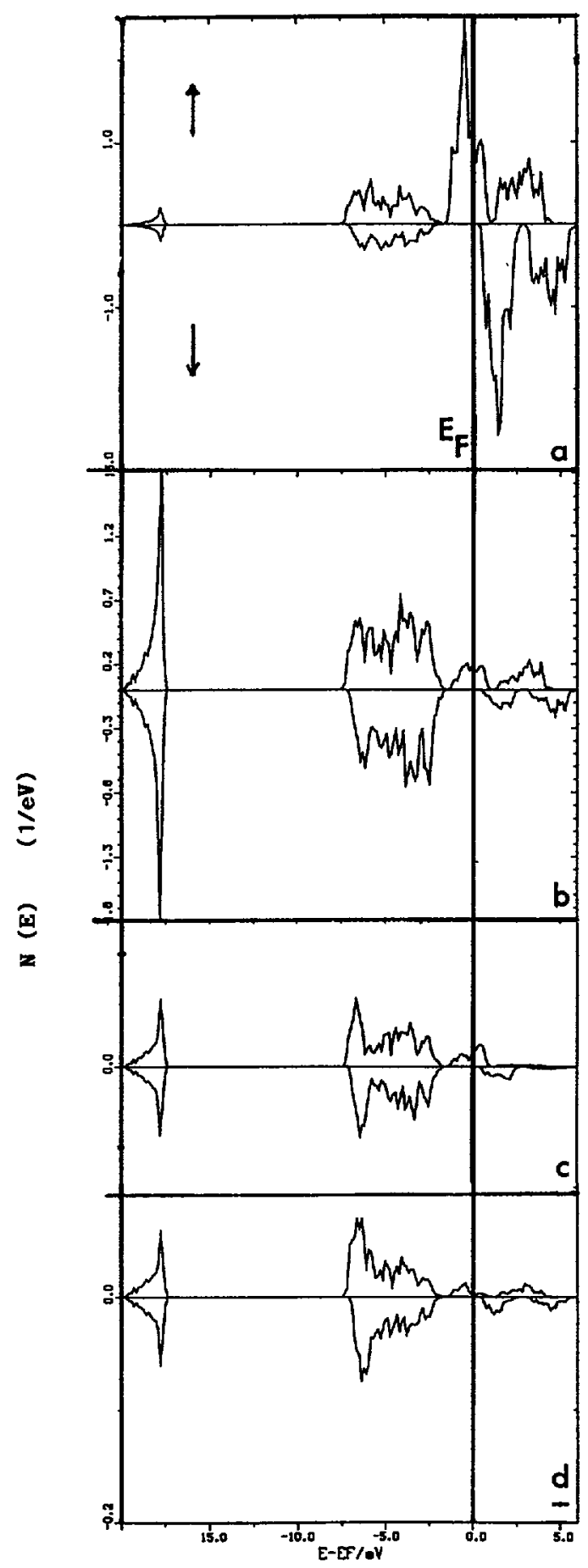

Fig. 5. - DOS de $\mathrm{CrO}_{2}$ projetées suivant les deux directions de spins sur les différents sites de la maille : a) $\mathrm{Cr}$, b) $\mathrm{O}$, c) $\mathrm{ES}_{1}$ et d) $\mathrm{ES}_{2}$.

[DOS of $\mathrm{CrO}_{2}$ projected along the 2 spin directions over the different sites of the lattice : a) $\mathrm{Cr}, \mathrm{b}$ ) $\mathrm{O}$, c) $\mathrm{ES}_{1}$ and d) $\mathrm{ES}_{2}$.] 


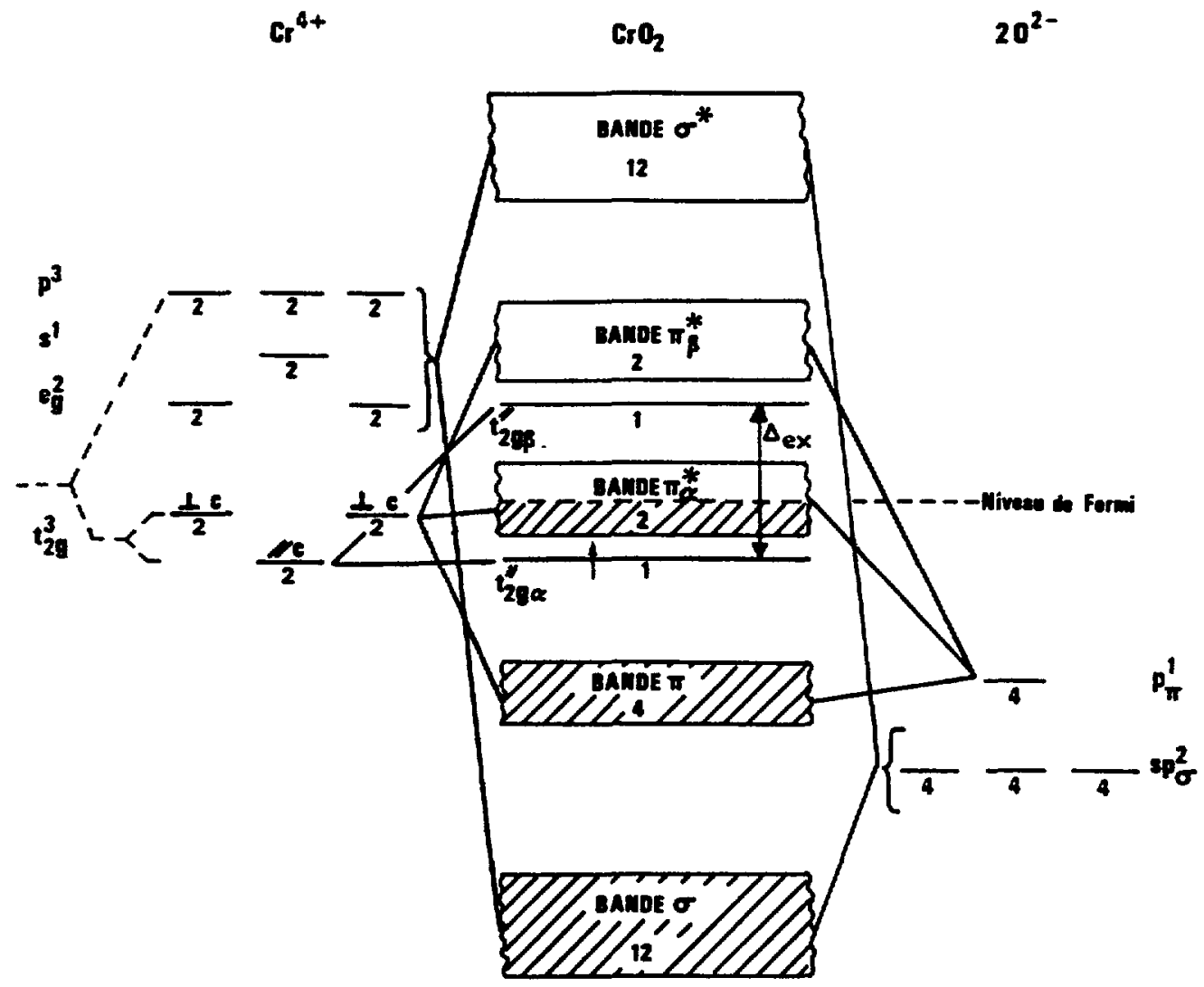

Fig. 6. - Diagramme de structure de bandes de $\mathrm{CrO}_{2}$ proposé par Goodenough [7].

[Band structure diagram of $\mathrm{CrO}_{2}$ as proposed by Goodenough [7].]

pour origine la polarisation des spins dans les sphères atomiques, notamment celle de $\mathrm{Cr}$. En effet la structure de bande de l'état non magnétique (i.e. sans polarisation des spins) de $\mathrm{CrO}_{2}$ donnée à la figure 7 montre un comportement intermédiaire car elle fait apparaître un gap d'amplitude voisine de celui de la figure $4 b$ entre les états $p$ de l'oxygène et $d$ du chrome et un comportement métallique (bande d de $\mathrm{Cr}$ traversée par $E_{\mathrm{F}}$ ) de l'oxyde.

4.3.2 Analyse des DOS. - L'examen des DOS (Fig. 5) permet de souligner un aspect qui est celui d'un mélange - ou hybridation - des différents états suivant les deux directions de spins pour $\mathrm{Cr}-3 \mathrm{~d}$ et $\mathrm{O}-2 \mathrm{p}$. Deux origines à ce mélange peuvent être proposées :

- un effet physique attribuable au caractère étendu des états $\pi$ (en termes de densité de charge) de l'oxygène à l'intérieur des sphères du chrome (Fig. 5a);

— un artefact introduit par le recouvrement des sphères atomiques (A.S.A.) (Fig. 5b).

5. Nouvelle approche de la structure de bande de $\mathrm{CrO}_{2}$.

Dans le premier paragraphe nous avons fait ressortir dans la littérature l'existence d'un double désaccord : celui entre les travaux expérimentaux [6] et [8] d'une part et celui entre ces derniers et les calculs de structure de bande [5] d'autre part. Alors que les études expérimentale [6] et théorique [5] semblent être en accord quant à la densité d'état finie à 


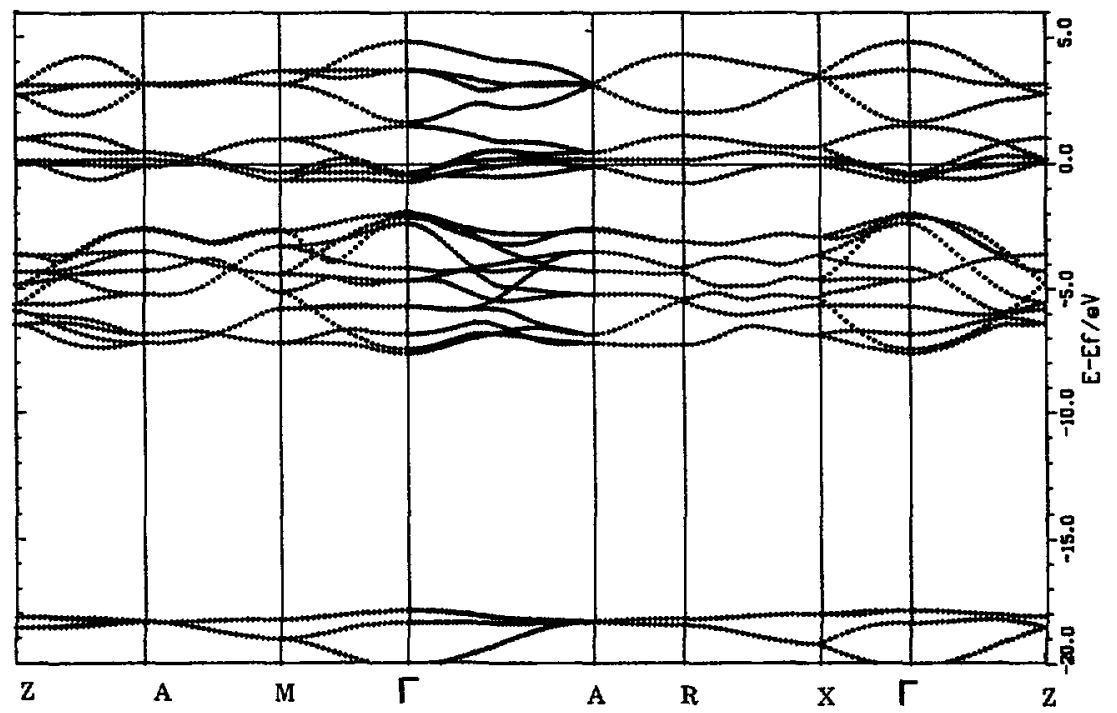

Fig. 7. - Structure de bandes de $\mathrm{CrO}_{2}$ dans un état non magnétique.

[Band structure of $\mathrm{CrO}_{2}$ in a non-magnetic state.]

$E_{\mathrm{F}}$, les résultats des études de couches minces de $\mathrm{CrO}_{2}$ par photoémission à résolution de spin [8] posent un problème quant à l'absence de densité d'états au niveau de Fermi.

Le calcul de la structure de bande de $\mathrm{CrO}_{2}$ [5] a été mené pour une seule valeur du paramètre $x_{4 \mathrm{f}}$ caractérisant la position de l'oxygène et par suite le type de déformation de l'octaèdre : soit pour un octaèdre allongé (pour $d_{1} / d_{2}>1$ ). Or le dépôt de couches minces peut engendrer des contraintes à l'interface substrat-couche mince et des défauts au niveau de la couche elle-même. Ces phénomènes seraient susceptibles d'induire un comportement différent de celui du matériau massique. Des couches contraintes ont été observées pour $\mathrm{SiO}_{2}$ sur substrat Si en microélectronique [14]. Ainsi l'étude de la structure électronique et magnétique de $\mathrm{CrO}_{2}$ a été entreprise suivant une nouvelle approche basée sur la déformation de l'octaèdre $\mathrm{CrO}_{6}$.

ETUDE DE L'INFLUENCE DE LA DÉFORMATION DE L'OCTAÈdRE CrO $_{6}$ SUR LA STRUCTURE DE BANDE DE $\mathrm{CrO}_{2}$. - Le modèle de structure de bande de Goodenough [7] suppose a priori un octaèdre $\mathrm{CrO}_{6}$ légèrement aplati. Aussi avons-nous adopté en première approche de faire varier progressivement la valeur de $x_{4 \mathrm{f}}$ caractérisant la position de l'oxygène dans le sens de l'affaissement de l'octaèdre $\mathrm{CrO}_{6}$. Afin d'observer l'unique effet de la variation de $x_{4 \mathrm{f}}$, les paramètres $a$ et $\frac{c}{a}$ ont été maintenus constants.

Des calculs effectués pour différentes valeurs de $x_{4 \mathrm{f}}$ deux résultats se dégagent :

i) Pour $0,29<x_{4 \mathrm{f}}<0,3053$ aucune variation des DOS par rapport à celles de la figure $5 \mathrm{ni}$ de l'aimantation n'ont été observées ;

ii) pour une valeur de $x$ de 0,2777 , la bande $3 \mathrm{~d}$ de $\mathrm{Cr}$ subit un éclatement au niveau de Fermi avec une importante diminution des DOS.

La figure 8 montre les DOS totales (a) et celles projetées au site du chrome (b) suivant les deux directions de spins. L'éclatement de la bande $3 \mathrm{~d}$ du chrome est clairement montré avec la nette diminution des DOS à $E_{\mathrm{F}}$. La déviation par rapport à la symétrie assimilée à celle d'un octaèdre caractéristique de la valeur $x=0,3053$ mène à un changement notable des DOS tant 


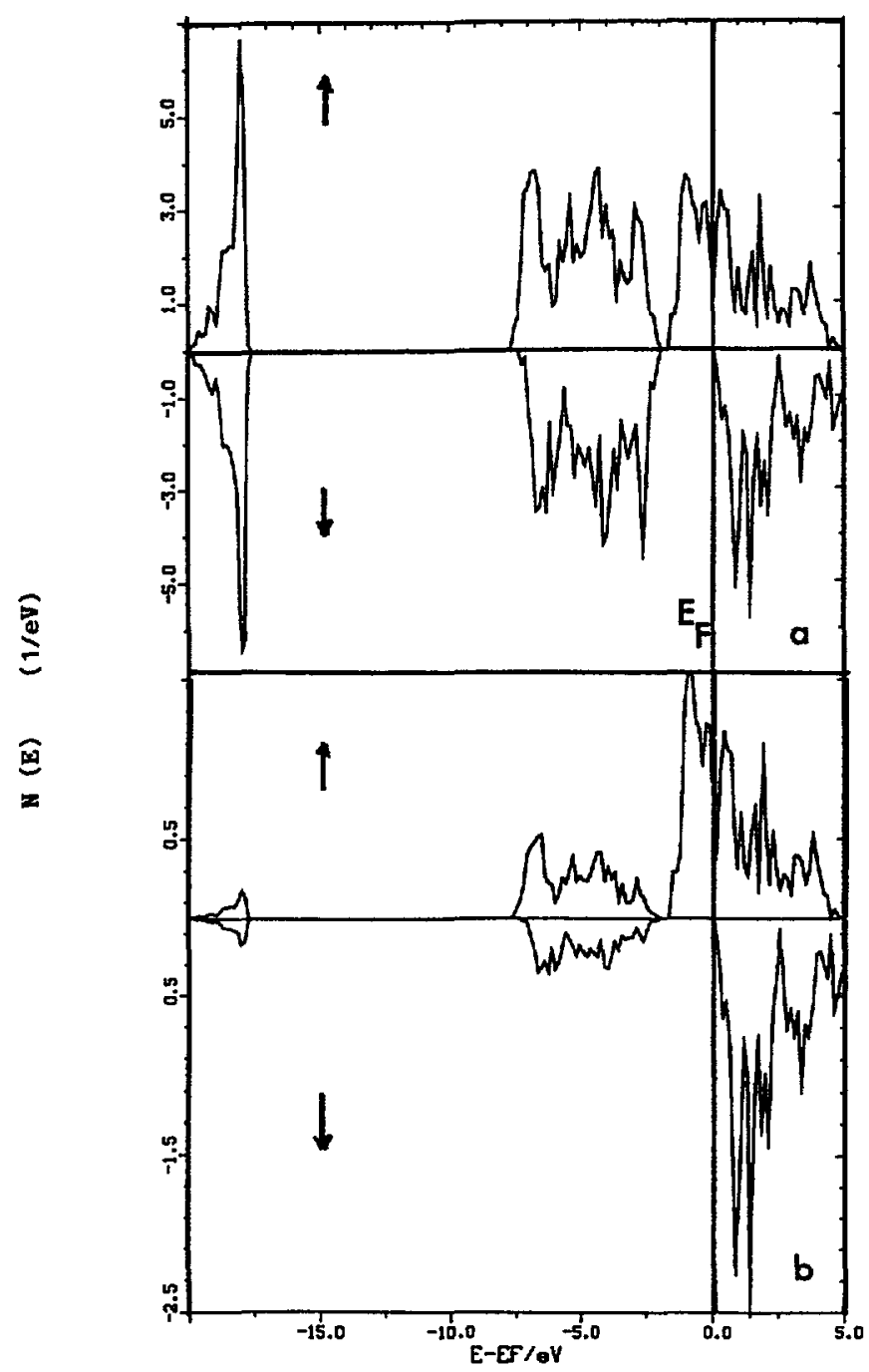

Fig. 8. - DOS de $\mathrm{CrO}_{2}$ pour un octaèdre comprimé $\left(x_{4 \mathrm{f}}=0,2777\right)$ montrant une diminution des densités d'états à $E_{\mathrm{F}}$ suite à l'éclatement de la bande $3 \mathrm{~d}$ du chrome. a) DOS totales ; b) DOS de Cr.

[DOS of $\mathrm{CrO}_{2}$ for a compressed octahedron $\left(x_{4 \mathrm{f}}=0.2777\right)$ showing their diminution at $E_{\mathrm{F}}$ consequently to a splitting of the $3 \mathrm{~d}$ band of chromium. a) Total DOS ; b) DOS of Cr.]

au niveau de la bande $d$ du chrome qu'au niveau des autres états ( $2 p$ et $2 s$ de l'oxygène). L'emplacement des diverses DOS reste le même et le gap dans les densités d'états des spins minoritaires est conservé. La diminution des DOS à $E_{\mathrm{F}}$ n'affecte pas les propriétés de l'oxyde qui resterait ferromagnétique et semi-métallique. Ces résultats obtenus pour un octaèdre $\mathrm{CrO}_{6}$ comprimé semblent corroborer en partie les travaux expérimentaux sur couches minces de $\mathrm{CrO}_{2}$ sans les confirmer complètement i.e. la faible DOS à $E_{\mathrm{F}}$ et $100 \%$ de polarisation des spins en dessous de $E_{\mathrm{F}}$. En fait Kämper et al. [8] commentent leur résultat de la faible densité d'états à $E_{\mathrm{F}}$ en émettant deux hypothèses : soit

1) l'ordre $t_{2 g}-e_{g}$ est inversé, soit

2) la bande $t_{2 g}$ doit éclater donnant un gap. 
La première hypothèse semble improbable étant donné la symétrie octaèdrique du site du chrome. La deuxième hypothèse nous paraît plus vraisemblable ; en outre elle serait illustrée par les calculs.

La déformation de l'octaèdre donnant ce comportement pourrait être inhérente à des contraintes créées dans le film de $\mathrm{CrO}_{2}$ lors de son élaboration. Il apparaît donc important de reprendre les manipulations de photoémission à polarisation de spin dans des conditions particulières de réalisation des couches minces.

Une élongation de l'octaèdre obtenue par une augmentation de la valeur de $x_{4 \mathrm{f}}$. soit $0.3053<x<0.34$ n'a pas permis de modifier le comportement de structure de bande de $\mathrm{CrO}_{2}$.

\section{Conclusions.}

La première partie de ce travail a permis de suggérer qu'une transition magnétique serait possible par compression sélective de la maille. Il est cependant difficile de prédire si cette transition aurait lieu avant une transformation allotropique sous pression. De telles études sont actuellement en cours sur des variétés hypothétiques de hautes pressions de $\mathrm{CrO}_{2}$ telles que la fluorine $\left(\mathrm{CaF}_{2}\right)$ ou encore $\mathrm{PbO}_{2}-\alpha$.

Dans la seconde partie une nouvelle approche de la structure électronique et magnétique de $\mathrm{CrO}_{2}$ a été abordée. Les calculs effectués pour une compression de l'octaèdre $\mathrm{CrO}_{6}$ au sein de la structure rutile ont permis de mettre en évidence l'éclatement de la bande $3 \mathrm{~d}$ du chrome. Ce résultat pourrait expliquer une faible densité d'états à $E_{\mathrm{F}}$.

\section{Remerciement.}

L'un de nous, S. Matar, remercie la fondation Alexander von Humboldt Stiftung pour une bourse d'études en Allemagne.

\section{Bibliographie}

[1] Michel A. et Bénard J., C.R. Acad. Sci. 200 (1935) 1316 ; Bull. Soc. Chim. Fr. 315 (1943).

[2] Swoboda T. J., Arthur Ir. P., Cox N. L., Ingraham J. N., Oppegard A. L. et Sadler M. S., J. Appl. Phys. 32 (1961) 3745.

[3] Demazeau G., Maestro P., Plante Th., Pouchard M. et Hagenmuller P., Ann. Chim. 3 (1978) 353.

[4] Mattheiss L. F., Phys. Rev. B 13 (1976) 2433.

[5] Schwarz K., J. Phys. F 16 (1986) L211.

[6] Ikemoto I., Ishil K., Kinoshita S., Kuroda H., Alario Franco M. A. et Thomas J. M., $J$. Solid State Chem. 17 (1976) 425.

[7] Goodenough J. B., Les oxydes des métaux de transition, Monographies de Chimie Minérale (Gauthier-Villars, Paris, 1973).

[8] Kamper K. P., Schmitt W., Guntherodt G., Gambino R. J. et Ruf R., Phys. Rev. Lett. 59 (1987) 2788.

[9] Thamer B. J., Douglas R. M. et Staritzky E., J. Am. Chem. Soc. 79 (1957) 547.

[10] Cloud W. H., Schreiber D. S. et Babcock K. R., J. Appl. Phys. 33 Suppl. (1962) 1193.

[11] Porta P., Marezio M., Remeika J. P. et Dernier P. D., Mater. Res. Bull. 7 (1972) 157.

[12] Williams A. R., Kúbler J. et Gelatt Jr. C. D., Phys. Rev. B 19 (1979) 6094.

[13] Koelling D. D. et Harmon B. N., J. Phys. C 10 (1977) 3107.

[14] Camelin Ch., Thèse Docteur, Université Bordeaux 1 (1985);

Camelin C., Demazeau G., Straboni A. et Buevoz J. L., Appl. Phys. Lett. 48 (1986) 1211. 